\title{
WEB APPLICATION BASED FILE TRANSFER IN CUSTOMIZED CLOUD
}

\author{
Teegala Srinivas \\ Student-(M.Tech.), CSE Dept., BVRIT College, A.P., INDIA
}

\begin{abstract}
Web Application based File Transfer app in a Customized Cloud is an open source and cloud web app based on the File Transfer system application which should be organized, designed by the app designer and developed by the app developer, the webapp based file transfer system which it runs on the open cloud web server with the graphical user based interface system. The user interface turns automatically the customized web application environment into file transfer system management system. It uses the cloud web directory automated services with the powerful management tools as the web file transfer with the multiple options in the admin dashboard interface. It allows to configure the custom designs. Web application users or the web app sites can be modified or updated according to the developer needs, so the web file transfer application visitors or the users can open the webapp accounts in the file transfer web application for creating the file transfer based user accounts. It can store or save the unlimited data without any bandwidth permissions. As the webapp administrator allows the web moderators to setup the individual user quotas and the customized quota for the portal users known as the quota system for webapp. The application administrators and the application moderators will get the full access to the webapp portal administration managing area and to the permissions to block the individual and the unknown users, webapp site ads can be embedded into the webapp site portal or the cloud file transfer website.
\end{abstract}

This application can also be converted into the customized individual mobile application such as for the various os- android, the windows phone etc. It allows the individual users or individual administrators to share their experience in the form of comments and the customized user article modules. Webapp portal enables to allow the various file formats where it can store the data files and other data on the file transfer oriented web-cloud. It's as fully loaded features with the directory service mode in order to optimize the custom data and to optimize the file transfer oriented cloud server optimization. Web application can also be deployed on any file transfer system. The web app can be installed on the various operating platform which it allows the administrators to embed the search engine into the Cloud File Transfer Web site.

\section{INTRODUCTION}

Open Source Web App based on the Cloud File Transfer System is a fully featured web application, it is very much easy to install the web app on the system, web application is manageable and the maintenance of web application or the file transfer web app site is possible.

It is the client dependent cloud file transfer based on the individual file sharing via network. Application runs as individually on private and public cloud file transfer sites and the public web file transferring system or the individual datafile sharing on the Web App Solution for both the Enterprise systems and the individual users.

Administrators will be able to create the ' $\mathrm{n}$ ' number of the different users where (:.n=Unlimited) and the Administrators will have full access to assign the customized permissions to the client user for one who registered manually on the cloud file transfer over internet network.

Users can also upload user data and can also download the user data files as the user needed, each user account and the every user account is mainly assigned with the different user quota settings and sometimes the user data upload limit is predefined or set by the individual administrators by default on the cloud web file transfer system.

Users can add the description and client users can update their account on the web app site by logging or by authenticating into the Cloud File Transfer System Site. Web app Cloud on file transfer (File Transfer system solution) also provides the advanced security with the SSL Socket Layers with both the security bit layers $128 \& 256$ bit, the individual users can do the upload for the multiple files securely and simultaneously at the same time on the site without any error.

Server always supports the individual users to upload the user unlimited data if upload data limit is not set manually by the admins (i.e., The Uploading Data Value is 0,0 is the Unlimited Data Access). This web application can also be hosted on the shared Linux cloud web hosting server. Uploading File extensions add-on supports the multiple file extensions to upload).

Whenever in the cloud the file transfer user logged into the web app site, Individual user will be redirected to the user dashboard web page which will have the organization information with the organization information and account 
details of the current user (i.e., Individual Client User). It uses the deep and advanced sorting list based on the various details of the user and displays along with the quota details of the individual user.

Cloud Web File Transfer Web app is designed and developed with the various versions, Versions can be updated at any time with the latest version of application whenever the latest update patch for app is available.

Web app file transfer system automatically synchronizes the system time and the date of the current file transfer cloud system. The records of the web application appusers will be automatically recorded individually and auto-saved into the client user account.

Web app site users can access the web cloud of file transfer system site virtually over the network via the portal, mobile devices access is possible with the help of cloud file transfer smartphone app. It also enables the users to predefine the functionality of adding mail accounts on the client user account.

Each and every cloud file remains as it is on the cloud file transfer of the Web server, web app client users can simultaneously access from the various web app browsers, Applicable for any browser on any device.

\section{SYSTEM REQUIREMENTS FOR THE WEB} APP FILE TRANSFER SYSTEM ON CLOUD

The customized Web Server on the web cloud should be installed with the Apache Web Server or IIS Webserver.

The current web cloud server must be enabled with the cloudserver add-ons with the fully qualified domain for web app.

The cloud based customized server should be organized with the active directory server and must be pre-configured with the admin defined settings.

The server should be installed with the database server i.e., MySQL Database app server with the completely configured web directory application server.

The computer or system should be installed and configured with the following of the Operating Systems such as Windows Operating System or Linux OS.

Web cloud with the following configured cloud server agents and the frontend and backend security/firewall in the cloud web server.
The security for web app server should be enabled by SSL for the fully qualified domain for Cloud Web App File Transfer site.

Should have continuous (i.e., 24x7) Internet Access without any bandwidth restrictions. Recommended with $1 \mathrm{Gbps}$ download link and 100Mbps Upload Link.

Preinstalled with the PHP 5.4 as minimum requirement

Advanced feature like ion Loaders is enabled and installed on the Cloud Web Server.

Cloud Server should be enabled with the GLibrary, PHP Memory of $128 \mathrm{MB}$ as minimum requirement, Multiple PHP Extensions and cloud server must be enabled with the XML Extensions.

\section{WEB FILE TRANSFER SYSTEM ON CUSTOMIZED CLOUD BASED SOLUTION}

Cloud Web based Web File Transfer System Web Application is the both public and private file transfer management portal which provides the universal cloud access to the client user data files over the web or intranet from the devices such as client user computer and the client handheld devices such as mobile devices and smartphones.

Cloud file transfer app also provides the standalone platform where the client users or subscribers can easily view and users can also synchronize the data uploaded by the user. Anonymous users will not have access to the file transfer web site. Users can also synchronize the bookmarks, details, date and contacts to the individual client user devices.

It also enables the customized cloud server to configure where the cloud app users can start editing right away from the various networks on the web. Custom cloud environment can be easily built by the developers, as it is open source to develop the code and enables to custom redesign the current web application according to the enterprise requirements and organization requirements.

In the current scenario, the file transfer web app makes the use of the cloud oriented web services and it allows the client users to share the organization confidential data with the other client users, affiliates and with the co-administrators. Users can synchronize the data according to the requirement on the customized cloud web app server.

Data can be easily shared among the personal devices such as Mobile devices, Handheld Devices, Smartphone Devices, Netbooks, Tablets and Personal Computers.

Using the web file transfer system client console, end-user data can be deployed on the embedded smartphone devices 
and it gives the ability to share the client user data from one device to another device in right time, It is scalable and highly secure from various malicious attacks and threats because it is pre-installed with the frontend security and the back end security, Which provides high security on the network in both the network areas such as public network area and the private network area on the internet, It's a fully custom controlled solution for the cloud data servers.

It enables the high security access with the various security permissions levels such as low security level, medium security level and the high security level. Whereas administrator will have access and permission to configure the security levels for the client user accounts according to the subscription levels in Web App site.

- It enables with the high user-data leakage web app protection, Cloud web app security, Cloud web app privacy and Cloud Web app Server policy to all the users.

- Outer side of the organization, unknown or the anonymous users cannot access the Cloud Storage File Transfer system on the Website.

- It can be deployed and run on self-oriented Web App hosting, Dedicated Web app Cloud Hosting and Shared Web app Cloud hosting to provide the Cloud File Transfer System solution which runs on the Customized Web app Cloud Server.

\section{ARCHITECTURE FOR WEBAPP}

\section{Architecure}

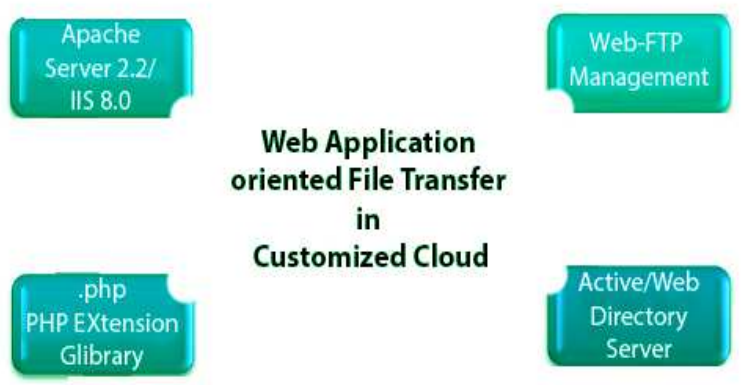

Fig-1: Architecture: Web Application based File Transfer in Customized Cloud

\section{CONCLUSIONS}

Web File Transfer System in the Customized Web Cloud is developed for the all the enterprises, educational \& Individual Institutions and also for testing developers. It is mainly deployed by the Cloud file Transfer System Service Providers. It's a fully organized and customized Web App Cloud File Transfer System Network Solution. Customized Cloud Web App with File Transfer Solution enables the API Acess to use as an individual application interface. It's a complete file transfer and storage solution for the entry level and the intermediate users, the data file storage web application site is mainly used for the high secure user-data processing and also to create the custom backups of the users on the Web App File System.

I hereby conclude that Web based File Transfer System (FTS) on custom cloud web application runs on the customized cloud web servers according to the user convenience, this application can be used commercially and personally. Hence it's a good web application for students and developers to develop more on the same application.

\section{REFERENCES}

[1]. LiutongXu,BoAi, "FTPGrid: A New Paradigm for Distributed FTP System" Published in year 2004,Vol3033, pp895 - 898.

[2]. SteffenHaak, StephanGrimm, "Towards Custom Cloud Services" Published in the year 2011, Vol6644, pp345-359.

[3]. Smith M D,RogerP., EdwardsR.N., MargaretJ.A., "Telnet and FTP: Remote Login and Retrieval of Information" Published in year 1997, pp25-29. 